\title{
Serum albumin levels and serum albumin-globulin ratio are associated with poor prognosis in glioblastoma
}

\author{
Kugeluke Yalikun ${ }^{1}$, Tuerhong Tuersun ${ }^{2}$, Maiaitituersun Abudula ${ }^{1}$, Maijudan Tiheiran ${ }^{3}$, Qiang Fu ${ }^{1}$, \\ Dilimulati Yisireyili ${ }^{1}$, Qingjiu Zhou ${ }^{1}$
}

${ }^{1}$ Department of Neurosurgery, ${ }^{2}$ Department of Intensive Care Unit, ${ }^{3}$ Department of Imaging, The First Affiliated Hospital of Xinjiang Medical University, Urumqi 830000, China

Contributions: (I) Conception and design: M Abudula, T Tuersun; (II) Administrative support: Q Fu; (III) Provision of study materials or patients: M Tiheiran; (IV) Collection and assembly of data: M Tiheiran, D Yisireyili; (V) Data analysis and interpretation: K Yalikun, Q Zhou; (VI) Manuscript writing: All authors; (VII) Final approval of manuscript: All authors.

Correspondence to: Qingjiu Zhou, MD. Department of Neurosurgery, The First Affiliated Hospital of Xinjiang Medical University, Liyushan South Road 137, Urumqi 830000, China. Email: 1210253194@qq.com.

Background: Serum albumin levels (ALB) and albumin-globulin ratio (AGR) are reliable and convenient
markers of the nutritional status and inflammation of human body, and ALB has been identified as a
prognostic factor in the patients of glioblastoma (GBM). However, no literature has reported the prediction
value of AGR for GBM.
Methods: In this study we evaluate the serum ALB and AGR levels for GBM. A total of 126 patients
with GBM who underwent surgical resection in our institution between 2013 and 2017 were analyzed
retrospectively. Clinical information was obtained from electronic medical records. Multiple logistic
regression and Cox proportional hazards models were used to assess the prediction value of preoperative
ALB and AGR for GBM.
Results: Preoperative ALB (HR 0.342, 95\% CI, 0.123-0.954, P=0.040) and postoperative adjuvant therapy
(HR 0.042, 95\% CI, 0.005-0.330, P=0.003) were significantly related to progression-free survival (PFS). Cox
regression analysis showed the significance of adjuvant therapy (HR 3.579, 95\% CI, 2.236-5.729, P $<0.001$ ).
Preoperative AGR (HR 0.280, 95\% CI, 0.103-0.763, P=0.013) and adjuvant therapy (HR 0.156, 95\% CI,
0.047-0.513, P=0.002) were showed significance, and Cox regression analysis showed preoperative AGR (HR
1.810, 95\% CI, 1.095-2.992, P=0.021) and adjuvant therapy (HR 4.702, 95\% CI, 2.841-7.782, P<0.001)
were independent predictors of overall survival (OS).

Conclusions: The ALB and AGR had significant predictive values for the prognosis of GBM; postoperative adjuvant treatment is also an independent predictor for the prognosis of GBM patients.

Keywords: Glioblastoma (GBM); albumin (ALB); albumin-globulin ratio (AGR); prognosis

Submitted Oct 29, 2019. Accepted for publication Jan 03, 2020.

doi: $10.21037 /$ tcr.2020.01.57

View this article at: http://dx.doi.org/10.21037/tcr.2020.01.57

\section{Introduction}

Glioma is the most common type of invasive malignant tumor in central nervous system (CNS), accounting for $65 \%$ of primary intracranial tumors $(1,2)$. In 2016, World Health Organization (WHO) classified glioma into grade I-IV histologically according to the degree of malignancy.
Grade IV is glioblastoma (GBM), which is highly invasive with poor prognosis (3). Despite the complete resection of GBM plus postoperative radiotherapy and chemotherapy, the median PFS and OS is not optimistic (4). Although certain prognostic factors for GBM have been reported $(5,6)$, the guiding function to the current treatment is limited, which warranted the new prognostic factors to be constantly 
studied.

ALB and the albumin-globulin ratio (AGR) can reflect the nutritional status of the human body, which is closely associated with immunosuppression (7). Previous studies showed that ALB and AGR can predict the prognosis of neoplasm $(8,9)$, besides, ALB can predict the prognosis of GBM (10-12), and there were few literatures had reported the prediction value of AGR on the prognosis of glioma (13). In this study, we aimed to evaluate the prognostic value of serum ALB and AGR in GBM by reviewing the relevant literatures.

\section{Methods}

\section{Study population}

The medical records of patients who underwent GBM resection at The First Affiliated Hospital of Xinjiang Medical University between January 2013 and December 2017 were reviewed. Patients were included on the basis of eligibility criteria, which were: (I) Diagnosis was confirmed by pathological examination after the first resection; (II) full pre-operative laboratory data were available (i.e., ALB, AGR); and (III) no hematological system disorders impaired liver function or primary tumor at other sites.

\section{Data collection}

Demographic information of patients, such as age, gender, nationality, pre-operative ABL, AGR, Karnofsky performance status (KPS), with or without any history of seizures, tumor site and maximum diameter of tumor, extent of surgical resection (total gross resection or incomplete resection), whether to receive postoperative adjuvant therapy, level of Ki-67 expression were collected from the medical records. Tumor characteristics were assessed using pre-operative magnetic resonance imaging (MRI). The extent of surgical resection was determined by the chief neurosurgeon during operation.

PFS was defined as the time interval between the date of surgery and the discovery of the first recurrence, or the time interval between the date of surgery and the death time of the patients without recurrence. Recurrence was defined as the appearance of new lesions at or outside the operative area during MRI scanning. OS was defined as the interval between date of the surgery and the date of death. Patients were censored at the end of follow-up, if the patient was still alive. The last follow-up was performed in December
2018. AGR was calculated as ALB count (g/L) divided by serum globulin count $(\mathrm{g} / \mathrm{L})$.

\section{Statistical analysis}

Descriptive analyses were used to describe patient demographics and clinical characteristics. Number of cases and percentages were calculated. We reported median PFS, 12-month PFS, also median OS, 18-month OS, and 95\% confidence intervals (CI) for each subgroup. Log-rank tests were used to compare ALB, AGR and other factors, $\mathrm{P}$ values were calculated. $\mathrm{P}$ values of $<0.05$ were considered statistically significant. The multiple logistic regression analysis was used for multivariate analysis, survival between items of statistical significance for log-rank tests, Cox proportional hazards method for evaluate the impact on survival. All variables (preoperative ALB and AGR, with or without any postoperative seizure, tumor site, degree of surgical resection, whether to receive postoperative adjuvant therapy and Ki-67 expression) were included in the final multivariate model based on a prior identification. Data management and statistical analyses were conducted using SPSS 20 software.

\section{Results}

One hundred and twenty-six adult patients with primary GBM met the inclusion criteria for the study. Median OS and median PFS were 10.8 and 5.7 months (Figure 1). Most of the patients were male $(59.5 \%)$, the median age was 55 years, (range, $19-80$ years), and the mean age was 55.2 years; $12.7 \%$ of the patients experienced preoperative seizures and $13.2 \%$ of the patients experienced postoperative seizures. The complete surgical resection of GBM was performed in $60.3 \%$ of the patients and postoperative adjuvant therapy was performed in $60.5 \%$ of the patients; $43.7 \%$ of the tumors were located in the right hemisphere of the brain, $46 \%$ in the left and $10.3 \%$ was located in both hemispheres, and $53.2 \%$ was large $(\geq 5 \mathrm{~cm})$. We also reported Ki-67 level of the tumor, and have shown that the patients with the Ki-67 expression outreached $30 \%$ was $51.6 \%$ (Table 1 ).

The median PFS and 12 -month PFS rate of the study sample were 5.7 months and $23.0 \%$, respectively (Figure 1A and Table 2). Preoperative ALB, postoperative seizure, adjuvant therapy and Ki-67 level were found to be significant by log-rank analysis $(\mathrm{P}<0.05)($ Table 2$)$. But preoperative ALB (HR 0.342, 95\% CI, 0.123-0.954, $\mathrm{P}=0.040$ ) and accepting adjuvant therapy after surgery 
Table 1 Patient characteristics

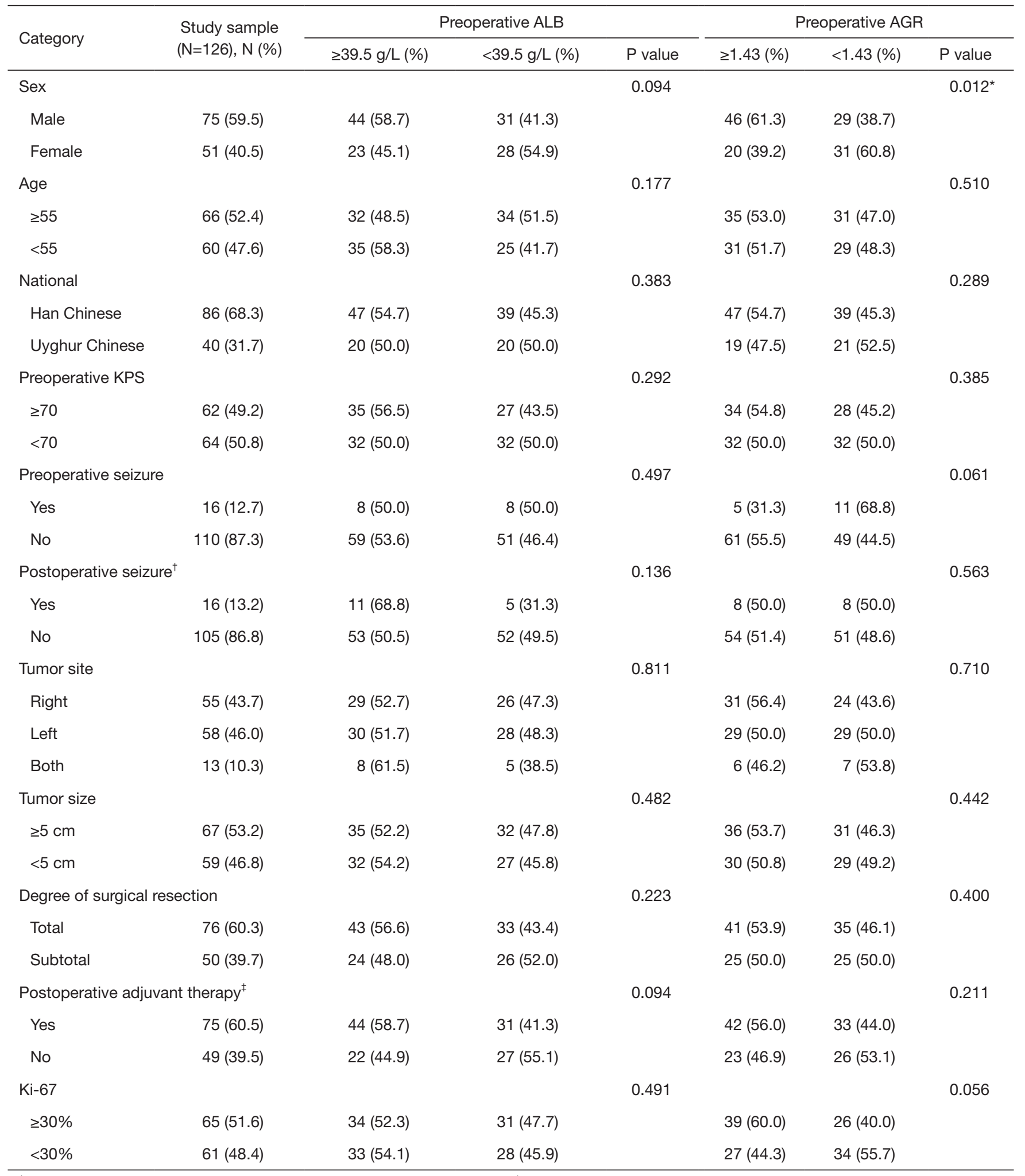

\footnotetext{
${ }^{\dagger}$, there were 5 case can't be confirmed whether seizure after surgery; ${ }^{\ddagger}$, there were 4 case can't be confirmed whether accept any adjuvant therapy after surgery. ${ }^{*}, \mathrm{P}<0.05$ showed statistically significant. ALB, serum albumin levels; AGR, albumin-globulin ratio; KPS, Karnofsky performance status.
} 

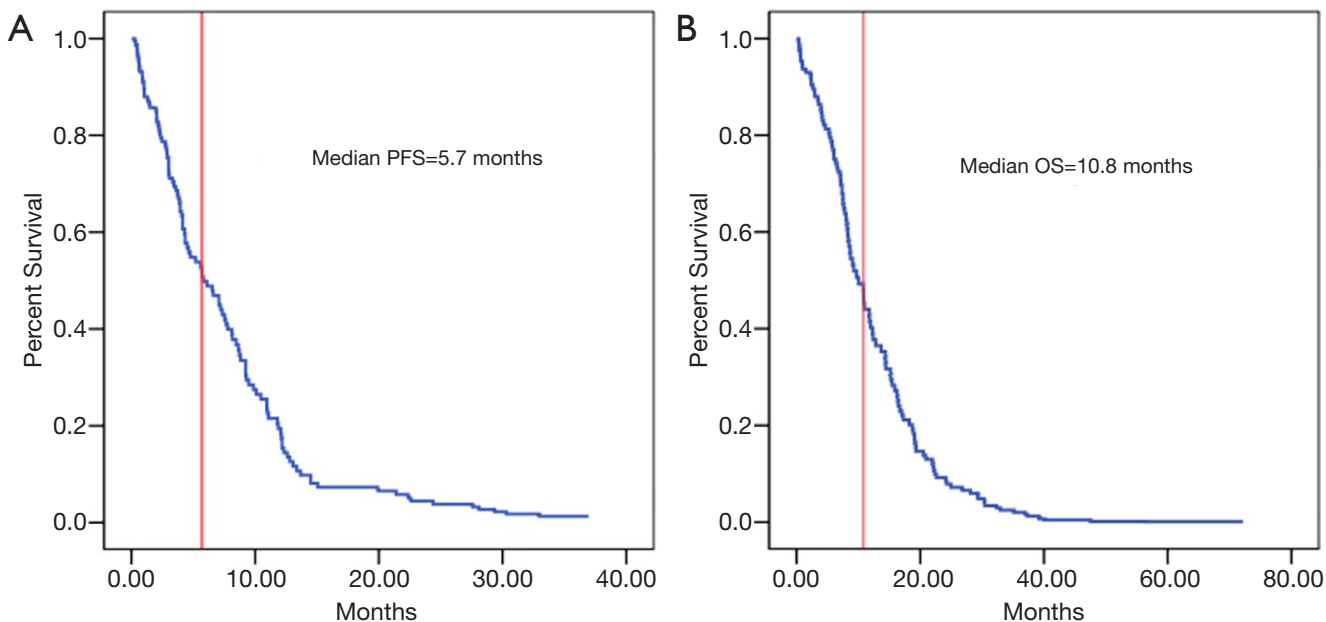

Figure 1 The percentage of PFS after the operation in the whole follow-up was showed in (A), and the median PFS was 5.7 months. While the percentage of OS after the operation in the whole follow-up was demonstrated in (B) with a median OS of 10.8 months. PFS, progression-free survival; OS, overall survival. The red line indicates the median.

(HR 0.042 , 95\% CI, 0.005-0.330, $\mathrm{P}=0.003$ ) were showed statistically significant by multivariate analysis (Table 3). Cox regression analysis showed that adjuvant therapy was statistically significant (HR 3.579, 95\% CI, 2.236-5.729) (Figure 2A), but preoperative ALB (HR 1.218, 95\% CI, $0.791-1.875, \mathrm{P}=0.371$ ) was not (Figure $2 B$ ).

The primary outcome of this study was death related to CNS tumor; deaths from other causes were considered as censored. Within this framework, the median OS and 18 -month OS rate of the study sample were 10.8 months (Figure 1B), 29.4\%, respectively (Table 3). Patients with different preoperative ALB and AGR levels experiencing seizure after surgery, tumor site and postoperative adjuvant therapy were found to be statistically significant $(\mathrm{P}<0.05)$ (Table 4). And those variables were included in a multivariate analysis, just the preoperative AGR (HR $0.280,95 \% \mathrm{CI}, 0.103-0.763, \mathrm{P}=0.013)$ and adjuvant therapy (HR 0.156, 95\% CI, 0.047-0.513, P=0.002) were found to be significant (Table 5). Cox regression analysis showed that preoperative AGR (HR 1.810, 95\% CI, 1.095-2.992, $\mathrm{P}=0.021$ ) (Figure 3A) and adjuvant therapy (HR 4.702, 95\% CI, 2.841-7.782, $\mathrm{P}<0.001$ ) (Figure 3B) were independent predictors of OS.

\section{Discussion}

GBM is the most common primary tumor of the CNS and its standard treatment is including surgical resection combined with radiotherapy and temozolomide (TMZ) chemotherapy (14). However, the survival of the patients with GBM varies and influenced by many factors. The fiveyear OS rate of patients is approximately $4.5 \%$ and the median survival of GBM is about 14.6 months, in spite of advanced diagnostic and therapeutic techniques (15).

In this study, we demonstrated that ALB and AGR are significantly independent prognostic factors in GBM. To our knowledge, ALB is one of the significant prognostic indicators of GBM, and the greater the ALB level is, the higher positive correlation with prognosis of GBM can be found, which indicates that elevated ALB serve as a protective role in survival of GBM patients. Our study showed ALB was an independent predictor of PFS in GBM (Tables 2,3), but it did not show statistical significances in the Cox analysis (Figure 2A). Univariate analysis showed ALB related with OS (Table 4), but multivariate analysis doesn't show any significance (Table 5). In oncology, nutrition supplies have focused great attention on cancer epidemiology, progression, and treatment outcomes (16). Several studies have suggested that nutrition condition is an essential factor for GBM patients' survival (17). Albumin and globulin are the main components of serum proteins, and albumin can reflect the nutrition status of the human body. Lower ALB has indicated poor nutrition status and had been reported to predict survival in various types of cancers (18-20). And increased ALB has been correlated with good clinical outcomes in GBM patients $(11,12)$. These data of previous studies were consistent with our research, and prolonged OS in GBM patients is importantly 
Table 2 Significant univariate predictors of progression-free survival in 126 patients with GBM

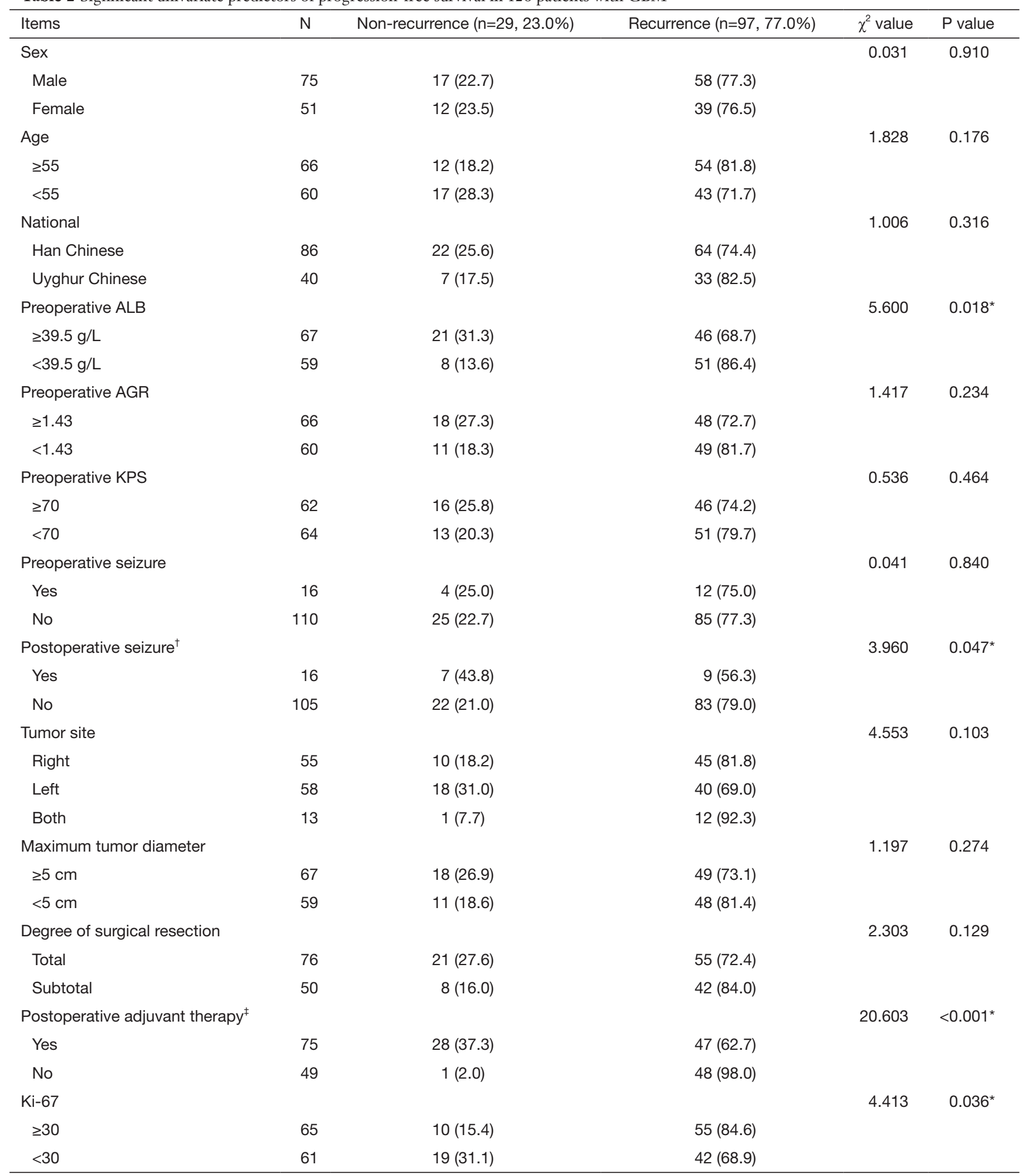

${ }^{\dagger}$, there were 5 case can’t be confirmed whether seizure after surgery; ${ }^{\ddagger}$, there were 4 case can't be confirmed whether accept any adjuvant therapy after surgery. *, P<0.05 showed statistically significant. GBM, glioblastoma; ALB, serum albumin levels; AGR, albumin-globulin ratio; KPS, Karnofsky performance status. 
Table 3 Significant multivariate predictors of progression-free survival in 126 patients with GBM

\begin{tabular}{lcccc}
\hline Variable & $\mathrm{B}$ & $\mathrm{P}$ value & $\mathrm{Exp}(\mathrm{B})$ & 0.342 \\
\hline Preoperative ALB & -1.073 & $0.040^{*}$ & $0.123-0.954$ \\
Postoperative seizure & -0.669 & 0.285 & 0.512 & $0.150-1.748$ \\
Postoperative adjuvant therapy & -3.17 & $0.003^{*}$ & 0.042 & $0.005-0.330$ \\
Ki-67 & -0.83 & 0.108 & 0.436 & $0.158-1.200$ \\
\hline
\end{tabular}

*, $\mathrm{P}<0.05$ showed statistically significant. GBM, glioblastoma; ALB, serum albumin levels.
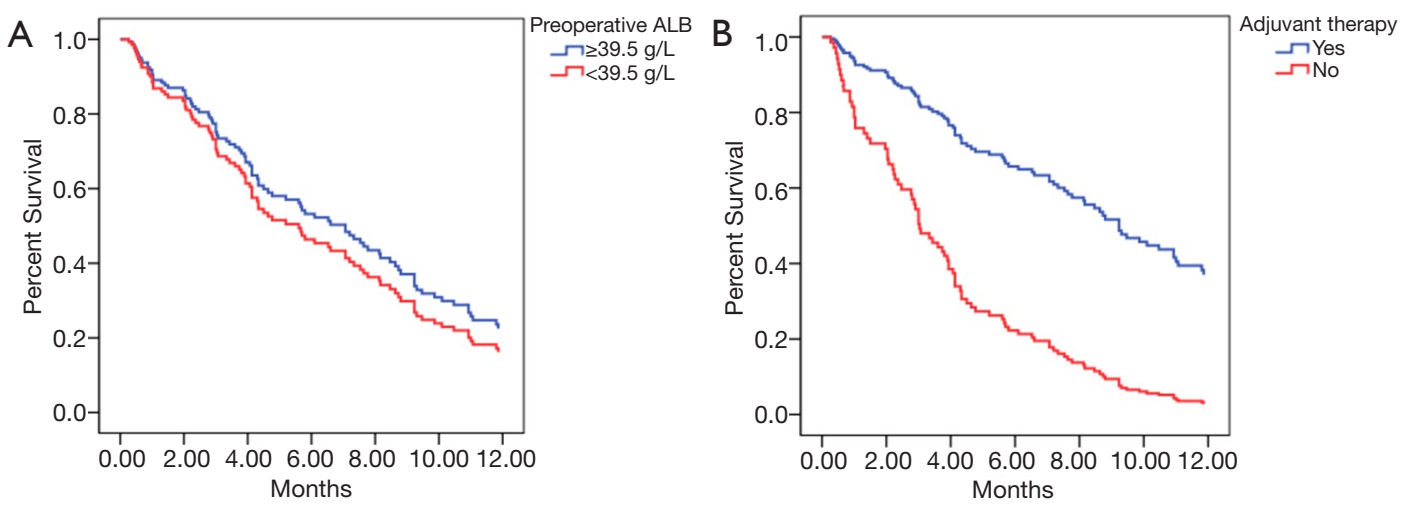

Figure 2 Cox regression analysis showed that the level of preoperative ALB was not related with PFS of patients with GBM (P=0.371) (A). Cox regression analysis showed that the PFS of patients with postoperative adjuvant therapy was longer than patients without postoperative adjuvant therapy $(\mathrm{P}<0.001)(B)$. PFS, progression-free survival; GBM, glioblastoma; ALB, Serum albumin levels.

due to high ALB level. Previous study has been proven ALB as a prognostic marker in GBM patients (11).

In recent years, several studies showed that AGR could be used to predict long-term mortality in various type of cancers $(21,22)$. And there is a small amount of studies on AGR in gliomas (13), while in our study, AGR is strongly correlated with prognosis of GBM, serum AGR $<1.43$ was associated with short OS of GBM (Table 5, Figure $3 A$ ) but no significance in PFS (Table 2). Recent Meta-analysis reported the outcomes of 4,136 patients from 14 available studies concluded that low AGR was significantly correlated with poor OS (HR 1.87, 95\% CI, 1.50-2.34, $\mathrm{P}<0.001)(23)$. Zhou et al. (24) suggested that small cell lung cancer patients with serum AGR $<1.29$ was 1.35 times more likely to die than those with AGR >1.29. Mao et al. (25) showed that patients with gastric cancer with AGR $<1.50$ had poor OS rate compared to those AGR $>1.50$. Wang et al. (26) reported that AGR $<1.51$ was associated with a significant deterioration of OS in the patients with early stage nonsmall cell lung cancer. In the study of Wang et al. (27), serum AGR $<1.45$ was an independent prognostic factor for poor PFS in patients with prostate cancer. For glioma, Liu et al. (13) suggested that preoperative AGR (AGR $>1.32$ ) indicated a better clinical prognosis and AGR was an independent prognostic factor for OS in patients with highgrade glioma. These results are strongly consistent with our retrospective data of GBM study.

The good prognosis with high ALB and AGR might be elucidated by the functions of nutrition factors including albumin and globulin. In our study, we analyzed the clinical outcomes of ALB and AGR in 126 patients with GBM, while among the several variables, low levels of ALB and AGR could reflect poor outcome of GBM patients. PFS and OS were extended with high ALB and AGR, indicating both are independent predictors of OS and PFS, besides, postoperative adjuvant therapy is significant prognostic factor for OS and PFS in GBM patients. So, ALB and AGR are convenient and inexpensive indices for predicting OS and PFS in GBM patients. Albumin has antioxidative effects anti-carcinogens and in cancer related inflammation, the albumin production also could be modulated by the proinflammatory cytokines released by tumor and immune cells 
Table 4 Significant univariate predictors in 126 patients with GBM during 18 months follow up

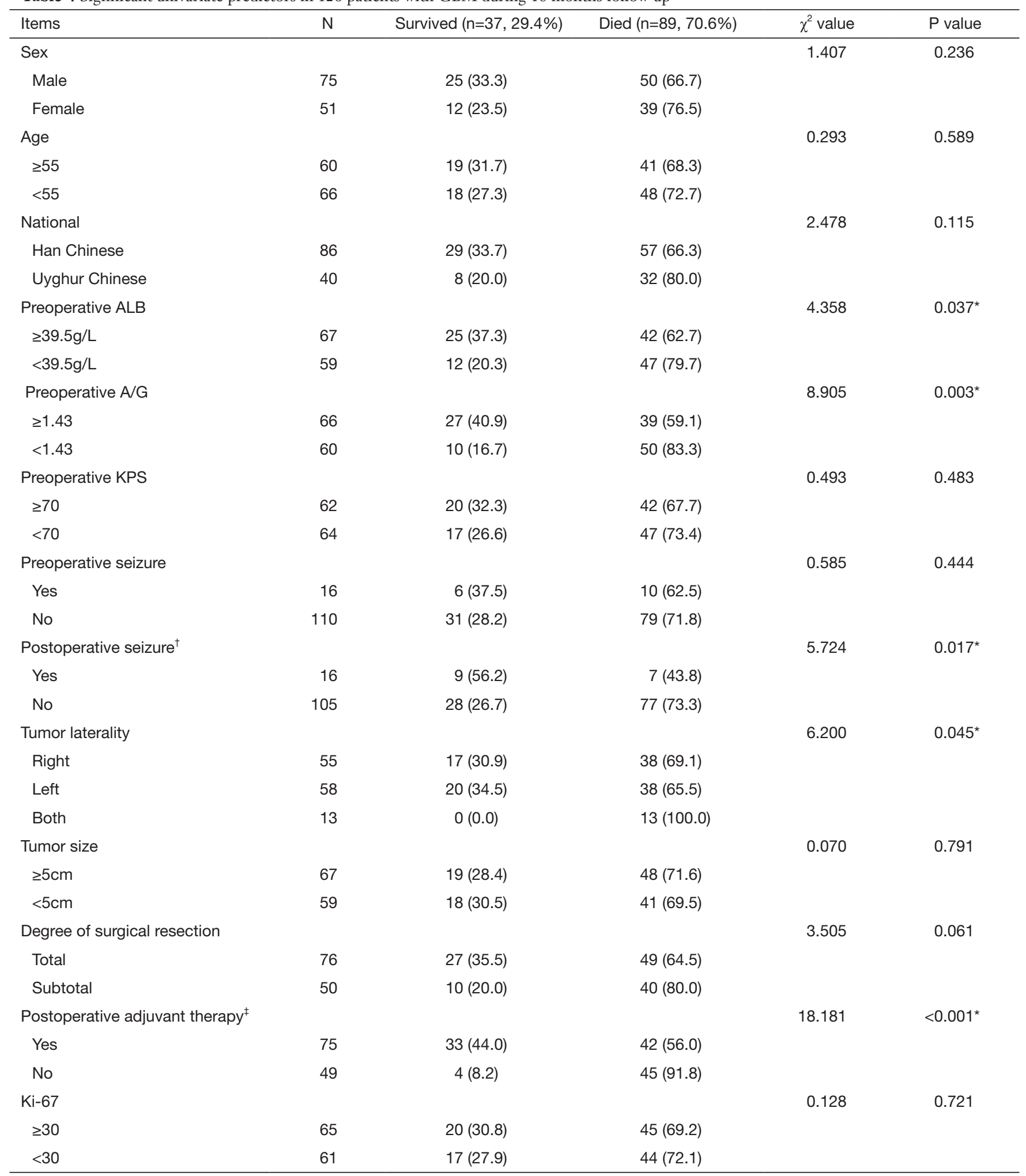

${ }^{\dagger}$, there were 5 case can't be confirmed whether seizure after surgery; ${ }^{\ddagger}$, there were 4 case can't be confirmed whether accept any adjuvant therapy after surgery. ${ }^{*}, \mathrm{P}<0.05$ showed statistically significant. GBM, glioblastoma; ALB, serum albumin levels; AGR, albumin-globulin ratio; KPS, Karnofsky performance status. 
Table 5 Significant multivariate predictors in 126 patients with GBM during 18months follow up

\begin{tabular}{lcccc}
\hline Characteristic & $\mathrm{B}$ & $\mathrm{P}$ value & $\mathrm{Exp}(\mathrm{B})$ & \multicolumn{2}{c}{$95 \% \mathrm{Cl}$} \\
\hline Preoperative ALB & -0.375 & 0.449 & 0.687 & $0.260-1.817$ \\
Preoperative AGR & -1.273 & $0.013^{*}$ & 0.280 & $0.103-0.763$ \\
Postoperative seizure & -0.872 & 0.155 & $-126-1.391$ \\
Tumor site & - & 0.896 & 0.156 & $0.047-0.513$ \\
Postoperative adjuvant therapy & -1.860 & $0.002^{*}$ & 0. \\
\hline
\end{tabular}

*, $\mathrm{P}<0.05$ showed statistically significant. GBM, glioblastoma; ALB, serum albumin levels; AGR, albumin-globulin ratio.
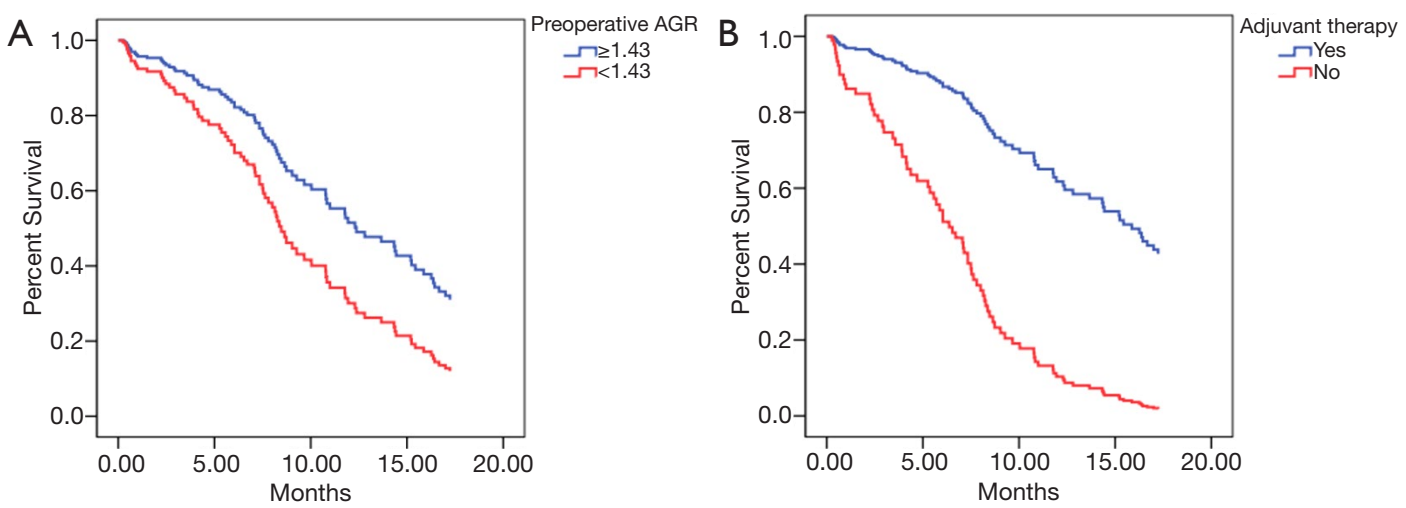

Figure 3 Cox regression analysis showed that the OS of patients with high preoperative AGR (AGR $\geq 1.43$ ) was longer than patients with low preoperative AGR (AGR <1.43) ( $\mathrm{P}=0.021)(\mathrm{A})$. Cox regression analysis showed that the OS of patients with postoperative adjuvant therapy was longer than patients without postoperative adjuvant therapy $(\mathrm{P}<0.001)(\mathrm{B})$. PFS, progression-free survival; ALB, serum albumin levels.

and serve as a role in cancer progression and angiogenesis $(28,29)$. In several analyses, all these nutritional indicators were associated with OS, and the prognostic effect of ALB can be attributed to its role as a nutritional indicator. Consistent with previous studies, our data showed that ALB has a good predictive value than other nutritional indicators in $\operatorname{GBM}(30,31)$. According to the results ALB and AGR are not only strong indicators of nutritional status, but also responsible for specific pathophysiological process such as inflammatory responses. The ALB and AGR may indicate the patients' general condition, nutritional statues and the severity of the inflammatory reactions.

Admittedly, there were some limitations in the present study. Our single center study had a small sample size with its retrospective nature, which may lead to some systematic bias.

\section{Conclusions}

Taken together, serum ALB and AGR are associated with poor prognosis in GBM, those are important nutritional factors of the human body, and however, the specific mechanism is still unclear and needs to be further investigated.

\section{Acknowledgments}

Funding: None.

\section{Footnote}

Conflicts of Interest: All authors have completed the ICMJE uniform disclosure form (available at http://dx.doi. org/10.21037/tcr.2020.01.57). The authors have no conflicts of interest to declare.

Ethical Statement: The authors are accountable for all aspects of the work in ensuring that questions related to the accuracy or integrity of any part of the work are 
appropriately investigated and resolved. The study was conducted in accordance with the Declaration of Helsinki (as revised in 2013). All procedures were approved by the ethics committees of The First Affiliated Hospital of Xinjiang Medical University based on the consent information of all families (No. 20180223-13) and informed consent was taken from all individual participants.

Open Access Statement: This is an Open Access article distributed in accordance with the Creative Commons Attribution-NonCommercial-NoDerivs 4.0 International License (CC BY-NC-ND 4.0), which permits the noncommercial replication and distribution of the article with the strict proviso that no changes or edits are made and the original work is properly cited (including links to both the formal publication through the relevant DOI and the license). See: https://creativecommons.org/licenses/by-nc-nd/4.0/.

\section{References}

1. Tang W, Fan W, Lau J, et al. Emerging blood-brainbarrier-crossing nanotechnology for brain cancer theranostics. Chem Soc Rev 2019;48:2967-3014.

2. Boussiotis VA, Charest A. Immunotherapies for malignant glioma. Oncogene 2018;37:1121-41.

3. Louis DN, Perry A, Reifenberger G. The 2016 World Health Organization Classification of Tumors of the Central Nervous System: A Summary. Acta Neuropathol 2016;131:803-20.

4. Weller M, Le Rhun E, Preusser M, et al. How we treat glioblastoma. ESMO Open 2019;4:e000520.

5. Silantyev AS, Falzone L, Libra M, et al. Current and Future Trends on Diagnosis and Prognosis of Glioblastoma: From Molecular Biology to Proteomics. Cells doi: 10.3390/cells8080863.

6. Liang TH, Kuo SH, Wang CW, et al. Adverse prognosis and distinct progression patterns after concurrent chemoradiotherapy for glioblastoma with synchronous subventricular zone and corpus callosum invasion. Radiother Oncol 2016;118:16-23.

7. Shoji F, Morodomi Y, Akamine T, et al. Predictive impact for postoperative recurrence using the preoperative prognostic nutritional index in pathological stage I nonsmall cell lung cancer. Lung Cancer 2016;98:15-21.

8. Oki S, Toiyama Y, Okugawa Y, et al. Clinical Burden of Preoperative Albumin-Globulin Ratio in Esophageal Cancer Patients. Am J Surg 2017;214:891-8.

9. Kühn T, Sookthai D, Graf ME, et al. Albumin, Bilirubin,
Uric Acid and Cancer Risk: Results From a Prospective Population-Based Study. Br J Cancer 2017;117:1572-9.

10. Schwartzbaum JA, Lal P, Evanoff W, et al. Presurgical serum albumin levels predict survival time from glioblastoma multiforme. J Neurooncol 1999;43:35-41.

11. Borg N, Guilfoyle MR, Greenberg DC, et al. Serum albumin and survival in glioblastoma multiforme. J Neurooncol 2011;105:77-81.

12. Han S, Huang Y, Li Z, et al. The prognostic role of preoperative serum albumin levels in glioblastoma patients. BMC Cancer 2015;15:108.

13. Liu J, Zhan YB, Zhang FJ, et al. Prognostic values of preoperative albumin to globulin ratio for predicting clinical outcome in patients with high-grade gliomas. Transl Cancer Res 2019;8:1727-33.

14. Nam JY, de Groot JF. Treatment of Glioblastoma. J Oncol Pract 2017;13:629-38.

15. Ostrom QT, Bauchet L, Davis FG, et al. The epidemiology of glioma in adults: a "state of the science" review. Neuro Oncol 2014;16:896-913.

16. Zitvogel L, Pietrocola F, Kroemer G. Nutrition, inflammation and cancer. Nat Immunol 2017;18:843-50.

17. Prieto I, Montemuiño S, Luna J, et al. The role of immunonutritional support in cancer treatment: Current evidence. Clin Nutr 2017;36:1457-64.

18. Ataseven B, du Bois A, Reinthaller A, et al. Preoperative serum albumin is associated with post-operative complication rate and overall survival in patients with epithelial ovarian cancer undergoing cytoreductive surgery. Gynecol Oncol 2015;138:560-5.

19. Chiang JM, Chang CJ, Jiang SF, et al. Pre-operative serum albumin level substantially predicts post-operative morbidity and mortality among patients with colorectal cancer who undergo elective colectomy. Eur J Cancer Care (Engl) 2017. doi: 10.1111/ecc.12403.

20. Ishizuka $M$, Nagata $H$, Takagi $K$, et al. Clinical Significance of the C-Reactive Protein to Albumin Ratio for Survival After Surgery for Colorectal Cancer. Ann Surg Oncol 2016;23:900-7.

21. Suh B, Park S, Shin DW, et al. Low Albumin-To-Globulin Ratio Associated With Cancer Incidence and Mortality in Generally Healthy Adults. Ann Oncol 2014;25:2260-6.

22. Deng Y, Pang Q, Miao RC, et al. Prognostic significance of pretreatment albumin/globulin ratio in patients with hepatocellular carcinoma. Onco Targets Ther 2016;9:5317-28.

23. Chi J, Xie Q, Jia J, et al. Prognostic Value of Albumin/ Globulin Ratio in Survival and Lymph Node Metastasis 
in Patients with Cancer: A Systematic Review and Metaanalysis. J Cancer 2018;9:2341-8.

24. Zhou T, He X, Fang W, et al. Pretreatment Albumin/ Globulin Ratio Predicts the Prognosis for Small-Cell Lung Cancer. Medicine (Baltimore) 2016;95:e3097.

25. Mao MJ, Wei XL, Sheng H, et al. Clinical Significance of Preoperative Albumin and Globulin Ratio in Patients with Gastric Cancer Undergoing Treatment. Biomed Res Int 2017;2017:3083267.

26. Wang Y, Li S, Hu X, et al. The prognostic value of serum albumin-globulin ratio in early-stage non-small cell lung cancer: a retrospective study. Cancer Manag Res 2019;11:3545-54.

27. Wang N, Liu JY, Li X, et al. Pretreatment Serum Albumin/ Globulin Ratio as a Prognostic Biomarker in Metastatic

Cite this article as: Yalikun $\mathrm{K}$, Tuersun $\mathrm{T}$, Abudula $\mathrm{M}$, Tiheiran M, Fu Q, Yisireyili D, Zhou Q. Serum albumin levels and serum albumin-globulin ratio are associated with poor prognosis in glioblastoma. Transl Cancer Res 2020;9(3):15941603. doi: $10.21037 /$ tcr.2020.01.57
Prostate Cancer Patients Treated With Maximal Androgen Blockade. Asian J Androl 2018;21:56-61.

28. Mantovani A, Allavena P, Sica A, et al. Cancer-related inflam-mation. Nature 2008;454:436-44.

29. Rothschild MA, Oratz M, Schreiber SS. Serum albumin. Hepatology 1988;8:385-401.

30. Lin MY, Liu WY, Tolan AM, et al. Preoperative serum albumin but not prealbumin is an excellent predictor of postoperative complications and mortality in patients with gastrointestinal cancer. Am Surg 2011;77:1286-9.

31. Fujii T, Sutoh T, Morita H, et al. Serum albumin is superior to prealbumin for predicting short-term recurrence in patients with operable colorectal cancer. Nutr Cancer 2012;64:1169-73. 